\title{
Diversity of bartonellae associated with small mammals inhabiting Free State province, South Africa
}

\author{
Correspondence \\ Richard J. Birtles \\ richard.birtles@liverpool.ac.uk
}

\author{
Anne-Marié Pretorius, ${ }^{1}$ Lorenza Beati ${ }^{2}$ and Richard J. Birtles ${ }^{3}$ \\ ${ }^{1}$ National Health Laboratory Service, Department of Medical Microbiology (G4), School \\ of Medicine, Faculty of Health Sciences, University of the Free State, PO Box 339, \\ Bloemfontein 9300, South Africa \\ 2Department of Epidemiology and Public Health, Yale University School of Medicine, PO Box \\ 208034, 60 College Street, New Haven, CT 06520-8034, USA \\ ${ }^{3}$ Centre for Comparative Infectious Diseases and Department of Veterinary Pathology, Faculty \\ of Veterinary Science, University of Liverpool, Neston CH64 7TE, UK
}

\section{INTRODUCTION}

Bartonella species are Gram-negative pleomorphic bacteria belonging to the $\alpha$-Proteobacteria that are able to invade and persist within erythrocytes and thus chronically infect the blood of their hosts. Transmission between hosts is primarily mediated by haematophagous arthropods (Breitschwerdt \& Kordick, 2000). Parasitism by bartonellae is widespread

Published online ahead of print on 16 April 2004 as DOI 10.1099/ ijs.0.03033-0.

Abbreviations: ML, maximum likelihood; MP, maximum parsimony; SA, South African.

The GenBank/EMBL/DDBJ accession numbers for the gltA sequences of the Bartonella isolates reported in this study are AJ583112AJ583136.

Transmission electron micrographs of cells of two novel isolates and a matrix of $g / t A$ dissimilarity scores are available as supplementary material in IJSEM Online. among mammals; natural infections are highly prevalent and have been reported in many different species of insectivores, lagomorphs, rodents, artiodactyls, carnivores and primates. Although the genus currently contains only 20 recognized taxa, a large number of other isolates, mostly isolated from rodent communities worldwide, have been partially characterized (Birtles et al., 1994; Kosoy et al., 1997; Ellis et al., 1999; Ying et al., 2002; Holmberg et al., 2003; Stevenson et al., 2003). Sequence data for almost 200 isolates have been deposited in GenBank and comparative analysis of these suggests that most isolates cannot be reasonably accommodated within the current classification.

As bartonellae are phenotypically similar, characterization and differentiation of species is extremely reliant on genotypic and phylogenetic methods. DNA-DNA hybridization has been used in the definition of most species, although relatedness values very close to the $70 \%$ threshold value have been reported (Kordick et al., 1996). The usefulness of 
comparative analysis of DNA sequence data to delineate Bartonella species has already been demonstrated (Lawson \& Collins, 1996), and the application of this approach as the primary criterion for species definition has recently been proposed (La Scola et al., 2003). Several genetic loci have been used for the characterization of Bartonella isolates (Houpikian \& Raoult, 2001), but comparative analysis of citrate synthase gene $(g l t A)$ fragments remains the most commonly used approach for identifying bartonellae and inferring evolutionary relationships within the genus (Birtles \& Raoult, 1996). Studies of inferred evolutionary relationships among Bartonella species have revealed that species parasitizing the same or similar host species are not necessarily closely related. However, phylogenetic reconstructions have demonstrated that clades comprising bartonellae isolated from species indigenous to Eurasia are divergent from clades containing isolates derived from animals indigenous to the Americas (Ellis et al., 1999; Bown et al., 2002).

Although much effort has been put into studying the biodiversity of bartonellae following their emergence as pathogens of humans and domesticated animals, fewer than 30 mammal species have been surveyed to date and thus it is likely that the true extent of variety within the genus is far larger than is currently recognized. In this study we have attempted to extend this exploration by studying bartonellae associated with small mammals endemic to South Africa. This is the first time that bartonellae associated with African wildlife have been examined and thus the results of this study should serve well in testing the validity of hypotheses concerning the basis of the divergence of bartonellae that have been derived from examination of isolates obtained from other continents.

\section{METHODS}

Sampling of animals. During 2000 and 2001, small terrestrial mammals were trapped live at sites in nine different Nature Reserves of the Free State province, South Africa (Table 1). Trapping sites were selected in an attempt to sample as wide a diversity of animals as possible. The trapping schedule at each site involved setting 50 strategically placed, baited, Sherman traps over four consecutive days, with traps being checked each morning and evening. Trapped animals were identified and euthanized and blood was collected aseptically by cardiac puncture and stored frozen at $-70{ }^{\circ} \mathrm{C}$.

Isolation of Bartonella species and phenotypic characterization. A $100 \mu \mathrm{l}$ aliquot of each blood sample was plated on to $10 \%$ horse blood-enriched Columbia agar. Plates were incubated at $35^{\circ} \mathrm{C}$ in $5 \% \mathrm{CO}_{2}$ for up to 45 days. Plates were checked daily for bacterial growth, and colonies tentatively identified as bartonellae (small, round and grey-white) were passaged on to fresh plates. Putative bartonellae were identified on the basis of their microscopic appearance following Gram staining and their lack of biochemical reactivity when tested using API 20E strips (bioMérieux). To assess their ultrastructure, some isolates were prepared for transmission electron microscopy using standard methods. Briefly, bacteria were suspended in $2 \%$ phosphotungstic acid $(\mathrm{pH} 7 \cdot 2)$, spread on a water surface and absorbed to Formvar-coated nickel grids. Samples were viewed under a Philips Tecnai 10 transmission electron microscope at $100 \mathrm{kV}$.

PCR and sequence analysis. Crude DNA extracts were obtained from isolates and a reference strain of Bartonella henselae (Houston$1^{\mathrm{T}}$ ) by boiling a heavy suspension of the organisms mixed $(4: 1)$ with a $20 \%(\mathrm{w} / \mathrm{v})$ solution of Chelex 100 in distilled water. PCRbased amplification of gltA fragments was performed as previously described (Birtles \& Raoult, 1996). Products were purified using a PCR Purification kit (Qiagen) and the nucleotide base sequence of each was determined commercially on both strands using the same primers as used in the PCR described above. For each amplification product, sequence data derived from each primer were compared, verified and combined using Align Plus 4 software (Scientific and Educational Software). Once ambiguities were resolved by repeating sequencing reactions, sequence similarity values between pairs of $698 \mathrm{bp}$ gltA sequences from which primer sequences had been removed were determined using the same software.

The 698 bp sequences were then manually aligned with homologous gltA fragments using MacClade (Sinauer Associates). As many of the gltA sequences derived from Bartonella species and strains deposited

Table 1. Survey of Bartonella species infecting rodent species collected from Free State nature reserves, South Africa

Species: 1, Aethomys namaquensis; 2, Mastomys natalensis; 3, Rhabdomys pumilio; 4, Otomys irroratus; 5, Pedetes capensis; 6, Malacothrix typica; 7, Rattus rattus; 8, Saccostomus campestris; 9, Suncus varilla; 10, Tatera leucogaster. For each rodent species at each location, the number of animals from which a Bartonella isolate was obtained is given, with the total number of animals trapped in parentheses.

\begin{tabular}{|c|c|c|c|c|c|c|c|c|c|c|c|c|}
\hline Nature reserve & Latitude and longitude & 1 & 2 & 3 & 4 & 5 & 6 & 7 & 8 & 9 & 10 & Total \\
\hline Koppies & $27^{\circ} 15^{\prime} \mathrm{S} 27^{\circ} 40^{\prime} \mathrm{E}$ & & $1(1)$ & & & & & & & & & $1(1)$ \\
\hline Sandveld & $27^{\circ} 40^{\prime}$ S $25^{\circ} 43^{\prime} \mathrm{E}$ & & $0(1)$ & & & $0(14)$ & & $0(1)$ & $0(1)$ & & $12(19)$ & $12(36)$ \\
\hline Soetdoring & $22^{\circ} 25^{\prime} \mathrm{S} 26^{\circ} 00^{\prime} \mathrm{E}$ & & $2(2)$ & & & & & & & & & $2(2)$ \\
\hline Caledon & $29^{\circ} 50^{\prime}$ S $27^{\circ} 40^{\prime} \mathrm{E}$ & $0(3)$ & $0(1)$ & $1(4)$ & & & $0(1)$ & & & & & $1(9)$ \\
\hline Tussen die Riviere & $30^{\circ} 30^{\prime} \mathrm{S} 26^{\circ} 07^{\prime} \mathrm{E}$ & $3(8)$ & $2(2)$ & & & & & & $1(1)$ & $0(2)$ & & $6(13)$ \\
\hline Naval Hill & $30^{\circ} 06^{\prime} \mathrm{S} 26^{\circ} 14^{\prime} \mathrm{E}$ & $3(3)$ & & & & & & & & & & $3(3)$ \\
\hline Total & & $11(19)$ & $9(15)$ & $4(9)$ & $1(2)$ & $0(16)$ & $0(1)$ & $0(1)$ & $1(2)$ & $0(2)$ & $12(19)$ & $38(86)$ \\
\hline
\end{tabular}


in GenBank were of a markedly shorter length, we also produced another data matrix (338 bp) to compare our data with all previously recognized Bartonella taxa. The two data matrices were analysed separately. Phylogenetic reconstructions were obtained by maximumparsimony (MP) and maximum-likelihood (ML) methods as implemented by PAUP (version 4.0 beta 8) (Sinauer Associates) and by Bayesian analysis by using MrBayes (version 2.01) (Huelsenbeck \& Ronquist, 2001; available at http://morphbank.ebc.uu.se/mrbayes/). MP heuristic searches for optimum trees were performed by swapping branches using the tree bisection reconnection algorithm, with all substitutions given equal weight. Branch support was assessed by 500 bootstrap replicates. The MP tree with the best likelihood score was used as the starting tree for the ML heuristic search. Nucleotide frequencies and transition/transversion ratios were estimated based on the MP tree via ML. Site-specific rates were estimated using a character partition by codon position. Bayesian Markov Monte Carlo analyses were performed by simultaneously running four independent chains for 500000 replicates and by using a general time-reversible model, with relative site rates estimated by codon position. Trees were sampled every 100 iterations for a total of 5000 samples. Trees saved before the likelihood values stabilized were discarded from the final sample. A $50 \%$ majority-rule consensus tree of the remaining trees was performed in PAUP and posterior probability values recorded for each branch.

Choice of sequences for inclusion in phylogenetic analyses. The $338 \mathrm{bp}$ alignment described above comprised 50 different sequences. These included 24 obtained from the South African (SA) rodents analysed in this study, 17 from representative strains of the Bartonella taxa with validly published names and nine from partially characterized Bartonella isolates. These nine were chosen either because they were found to share relatively high levels of sequence similarity with one or more of the sequences obtained from SA rodents (C5-rat and MR1) or because they were representatives of previously identified phylogenetic clades within the genus Bartonella; the provenance of R-phy2, C1-phy and R-phy1 has been detailed by Birtles et al. (1999) and their phylogeny explored in a study by Birtles \& Raoult (1996), whereas the provenance and phylogenies of SH6397GA, SH6396GA, SH6537GA and PL7238NC have been detailed by Kosoy et al. (1997). The 698 bp alignment comprised 35 different sequences. Twenty-nine of these sequences belonged to the SA rodents or to other Bartonella taxa identified as sharing a specific relationship with the SA rodent strains after phylogenetic analysis of the $338 \mathrm{bp}$ alignment. The remaining six sequences were included as outliers, against which the phylogeny inferred among the SA rodent isolates and their immediate sister taxa could be referred. The accession numbers for all sequences used in this study are given in Table 2 or adjacent to the Bartonella species name and/or strain designation in Fig. 1.

Calculation of frequency of inter-gltA sequence dissimilarity values. This work was attempted as an alternative approach to clarifying taxonomic relationships among the bartonellae recovered from SA rodents and other closely related isolates, and with the additional aim of further assessing the applicability of recently proposed criteria for the delineation of Bartonella taxa using gene sequence similarity values (La Scola et al., 2003). Pairwise comparisons were determined among 29 partial (698 bp) gltA sequences obtained from members of the phylogenetically defined clusters I and II (see Fig. 2) using Align Plus 4. These clusters contained all SA rodent-associated isolates ( 25 sequences) and four other bartonellae (Bartonella elizabethae, Bartonella grahamii, C5rat and MR1). A total of 406 intersequence dissimilarity scores were obtained from these pairwise comparisons. The frequency of individual scores among these 406 was calculated and the distribution of frequencies examined.

\section{RESULTS}

\section{Details of trapped rodents and culture success}

A total of 86 animals were trapped during the study (Table 1). Overall trapping success was about $5 \%$ and ranged from $0.5 \%$ at Koppies to $18 \%$ at Sandveld, suggesting that rodent abundance varied widely between sites. A total of 10 different species of small mammal were trapped. However, as $78(91 \%)$ of the animals belonged to five species, the remaining five species were each represented by only one or two individuals. The richness of trapped species also varied between sites, although this parameter was likely influenced by rodent abundance. Four sites, at all of which trapping success had been $<1 \%$, yielded only one species. Between three and five species were trapped at the remaining five sites. The distribution of small mammal species also varied; five species were trapped exclusively at one site, although four of these were among those species represented by two or fewer representatives. Of the small mammals trapped in reasonable numbers, the Natal multimammate mouse, Mastomys natalensis, was found at seven sites, but the bushveld gerbil, Tatera leucogaster, was found at only one. Before euthanasia, all animals appeared healthy with no gross abnormalities.

The 86 blood samples yielded 38 cultures of putative bartonellae within 2 weeks of incubation. Colonial morphology was typical of primary Bartonella cultures, with small, off-white colonies appearing firm, dry and embedded. The number of c.f.u. varied from fewer than 10 to 'thousands' (per $100 \mu \mathrm{l}$ sample). Subsequent passage was achieved for 32 of the 38 isolates (Table 2). The isolates lost were three obtained from Aethomys namaquensis trapped at Iona, one obtained from an $M$. natalensis trapped at Iona, one obtained from a Rhabdomys pumilio trapped at Caledon and one obtained from an Otomys irroratus (vlei rat) trapped at Gariep. All 32 successfully passaged isolates were confirmed as bartonellae on the basis of their microscopic appearance as weakly staining, Gram-negative, small, pleomorphic cocco-bacilli and on their biochemical inactivity when tested using an API 20E strip. Electron microscopy was performed on three isolates, AN-tr103, MN-ga6 and RP-io114 (chosen on the basis of their gltA sequence variation). Examination of electron micrographs revealed that all three isolates possess a typical Gramnegative, rod-shaped morphology. All three also appeared to possess lophotrichous flagella-like appendages and AN-tr103 possessed substantial polar tufts of pili-like appendages (see supplementary figures available in IJSEM Online).

The overall prevalence of infection was $44 \%$. No evidence of infection was found in three of the poorly represented small mammal species and, perhaps more interestingly, in the springhare, Pedetes capensis, for which 16 animals were tested (Table 1). All animals yielding bartonellae were, therefore, members of the rodent family Muridae. Among 
Table 2. Provenance of the 32 successfully passaged Bartonella isolates

Common names of the host species are Namaqua rock mouse (Aethomys namaquensis), striped mouse (Rhabdomys pumilio), Natal multimammate mouse (Mastomys natalensis), bushveld gerbil (Tatera leucogaster) and pouched mouse (Saccostomus campestris).

\begin{tabular}{|c|c|c|c|}
\hline Isolate & Host species & Trap location & $\begin{array}{c}\text { GenBank accession } \\
\text { no. }(\text { glt } A)\end{array}$ \\
\hline AN-nh1 & Aethomys namaquensis & Naval Hill & AJ583112 \\
\hline AN-nh2 & A. namaquensis & Naval Hill & AJ583113 \\
\hline AN-nh3 & A. namaquensis & Naval Hill & AJ583114 \\
\hline AN-tr1 & A. namaquensis & Tussen die Riviere & AJ583115 \\
\hline AN-tr2 & A. namaquensis & Tussen die Riviere & AJ583116 \\
\hline AN-iol & A. namaquensis & Iona & Identical to $\mathrm{AN}-\mathrm{nh} 1^{*}$ \\
\hline AN-io2 & A. namaquensis & Iona & AJ583117 \\
\hline AN-tr103 & A. namaquensis & Tussen die Riviere & AJ583118 \\
\hline RP-ga109 & Rhabdomys pumilio & Gariep & AJ583119 \\
\hline RP-io111 & R. pumilio & Iona & AJ583120 \\
\hline RP-io114 & R. pumilio & Iona & AJ583121 \\
\hline MN-tr1 & Mastomys natalensis & Tussen die Riviere & AJ583122 \\
\hline MN-sol & M. natalensis & Soetdoring & AJ583123 \\
\hline MN-so2 & M. natalensis & Soetdoring & Identical to $\mathrm{MN}$-sol \\
\hline MN-ko1 & M. natalensis & Koppies & AJ583124 \\
\hline MN-tr4 & M. natalensis & Tussen die Riviere & AJ583125 \\
\hline MN-ga6 & M. natalensis & Gariep & AJ583126 \\
\hline MN-ga8 & M. natalensis & Gariep & AJ583127 \\
\hline MN-ga18 & M. natalensis & Gariep & AJ583128 \\
\hline TL-sv1 & Tatera leucogaster & Sandveld & AJ583129 \\
\hline TL-sv4 & T. leucogaster & Sandveld & AJ583130 \\
\hline TL-sv6 & T. leucogaster & Sandveld & AJ583131 \\
\hline TL-sv2 & T. leucogaster & Sandveld & AJ583133 \\
\hline TL-sv3 & T. leucogaster & Sandveld & AJ583134 \\
\hline TL-sv5 & T. leucogaster & Sandveld & Identical to $\mathrm{MN}$-so1 \\
\hline TL-sv7 & T. leucogaster & Sandveld & AJ583135 \\
\hline TL-sv8 & T. leucogaster & Sandveld & Identical to TL-sv1 \\
\hline TL-sv9 & T. leucogaster & Sandveld & AJ583136 \\
\hline TL-sv10 & T. leucogaster & Sandveld & Identical to TL-sv9 \\
\hline TL-sv15 & T. leucogaster & Sandveld & Identical to TL-sv1 \\
\hline TL-sv16 & T. leucogaster & Sandveld & Identical to $\mathrm{MN}$-sol \\
\hline SC-tr1 & Saccostomus campestris & Tussen die Riviere & AJ583132 \\
\hline
\end{tabular}

${ }^{\star}$ Sequences found to be identical to others encountered earlier in the study were not submitted to GenBank.

the well-represented species, infection prevalences were similar, ranging from $44 \%$ in the striped mouse, R. pumilio, to $63 \%$ in T. leucogaster (Table 1). Overall infection prevalence and host-specific infection prevalences also varied among sites (Table 1).

\section{gltA sequence analysis}

Partial gltA fragments of $698 \mathrm{bp}$ were obtained for all 32 successfully passaged isolates. Two sets of comparative sequence analyses were performed on these new data. First, all sequences were shortened to $338 \mathrm{bp}$ to allow their comparison with those available for all previously characterized Bartonella species (as available data for some of these species were limited to this length of sequence). Comparison of these data demonstrated that the isolates yielded 24 different sequences, all of which were distinct from the gltA sequences of the recognized Bartonella species or other partially characterized strains. The gltA sequences most similar to those of the SA rodent-associated bartonellae were those of B. elizabethae, B. grahamii and Bartonella tribocorum, but these were at least $4 \%$ dissimilar.

The second set of comparative sequence analyses was performed using full-length (698 bp) gltA fragments from the SA rodent-associated isolates together with those available from other Bartonella species and strains. Unsurprisingly, some of the full-length sequences were indistinguishable 


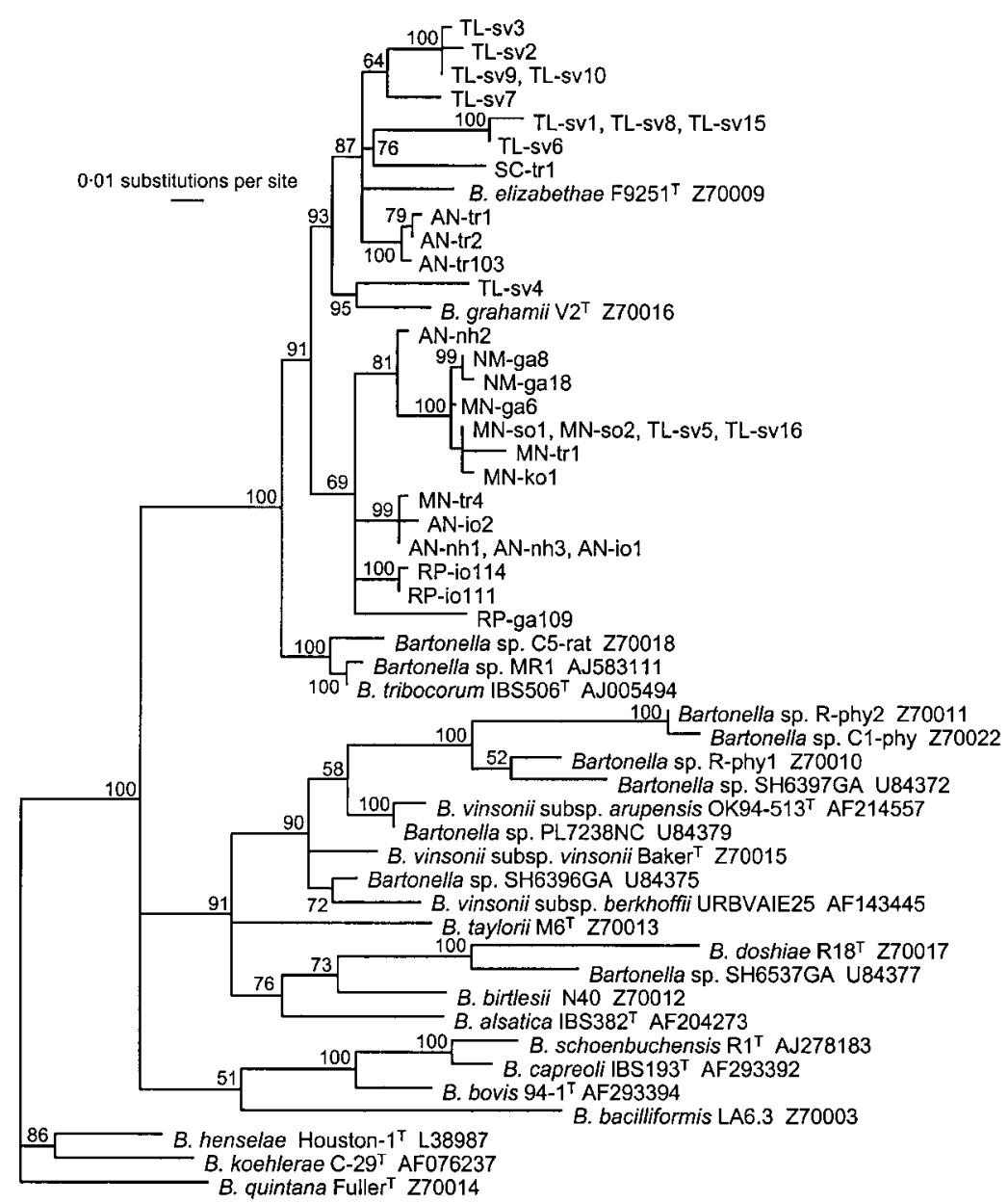

Fig. 1. Phylogenetic tree inferred using a Bayesian approach from an alignment of $338 \mathrm{bp}$ gltA fragments obtained from the SA rodent-associated bartonellae, representatives of the established Bartonella taxa and other partially characterized Bartonella isolates. Significant bootstrap values are indicated at relevant nodes. from one another. Sequences from isolates TL-sv1, TL-sv8 and TL-sv15 were the same as one another, as were those from isolates TL-sv9 and TL-sv10, isolates TL-sv5, TL-sv16, $\mathrm{MN}-$ sol and $\mathrm{MN}$-so2, and isolates $\mathrm{AN}-\mathrm{nh} 1$ and $\mathrm{AN}-\mathrm{io} 1$ (Table 2). Thus, 25 different sequences were obtained; the gltA sequences of isolates AN-nh1 and AN-nh3, which were identical when compared over 338 bp (above), differed when compared over $698 \mathrm{bp}$. None of the 25 sequences were identical to any other gltA sequence in GenBank and, as observed for the 338 bp sequences, they shared highest similarity $(96 \%)$ with 698 bp gltA fragments of $B$. elizabethae and B. grahamii. However, among the different gltA sequences obtained from the SA rodent-associated bartonellae, similarity values ranged from 91 to $99 \%$.

\section{Phylogeny}

The 338 bp alignment contained 105 parsimony informative characters. The MP heuristic search recovered a single most parsimonious tree (length $444 ; \mathrm{CI}=0 \cdot 480$; $\mathrm{RI}=0 \cdot 687$; not shown). The Bayesian $50 \%$ majority rule consensus tree of the 4800 retained samples is shown in Fig. 1, from which it was apparent that the SA rodent-associated bartonellae shared a strongly supported, discrete cluster within the radius of the genus Bartonella with the species $B$. grahamii, B. elizabethae and B. tribocorum.

The 698 bp alignment contained a total of 160 parsimony informative characters. The MP heuristic search recovered a single most parsimonious tree (length $=474 ; \mathrm{CI}=0.605$; $\mathrm{RI}=0 \cdot 790$ ) (Fig. 2). Isolates R-phy2, R-phy1, C1-phy and Bartonella vinsonii subsp. arupensis, B. vinsonii subsp. vinsonii and Bartonella taylorii were used as outgroups, because bootstrapping separated these sequences from all the others in $100 \%$ of the replicates. The ML heuristic search was completed after 225368 rearrangements and produced a total of $16 \mathrm{ML}$ trees. Because the topologies of the ML tree with the best ML score $(-\ln L=3377 \cdot 44)$ and of the Bayesian $50 \%$ majority-rule consensus tree were perfectly identical, we have only shown the Bayesian reconstruction (Fig. 2). This tree was obtained after the 130 trees, sampled before the likelihood values became stable, were eliminated.

The architecture of the MP and Bayesian-derived reconstructions presented in Fig. 2 were very similar to one another and to those inferred from the $338 \mathrm{bp}$ alignment (Fig. 1). Indeed, in Fig. 2, the clusters resolved by the two methods of analysis were the same with the exception of 


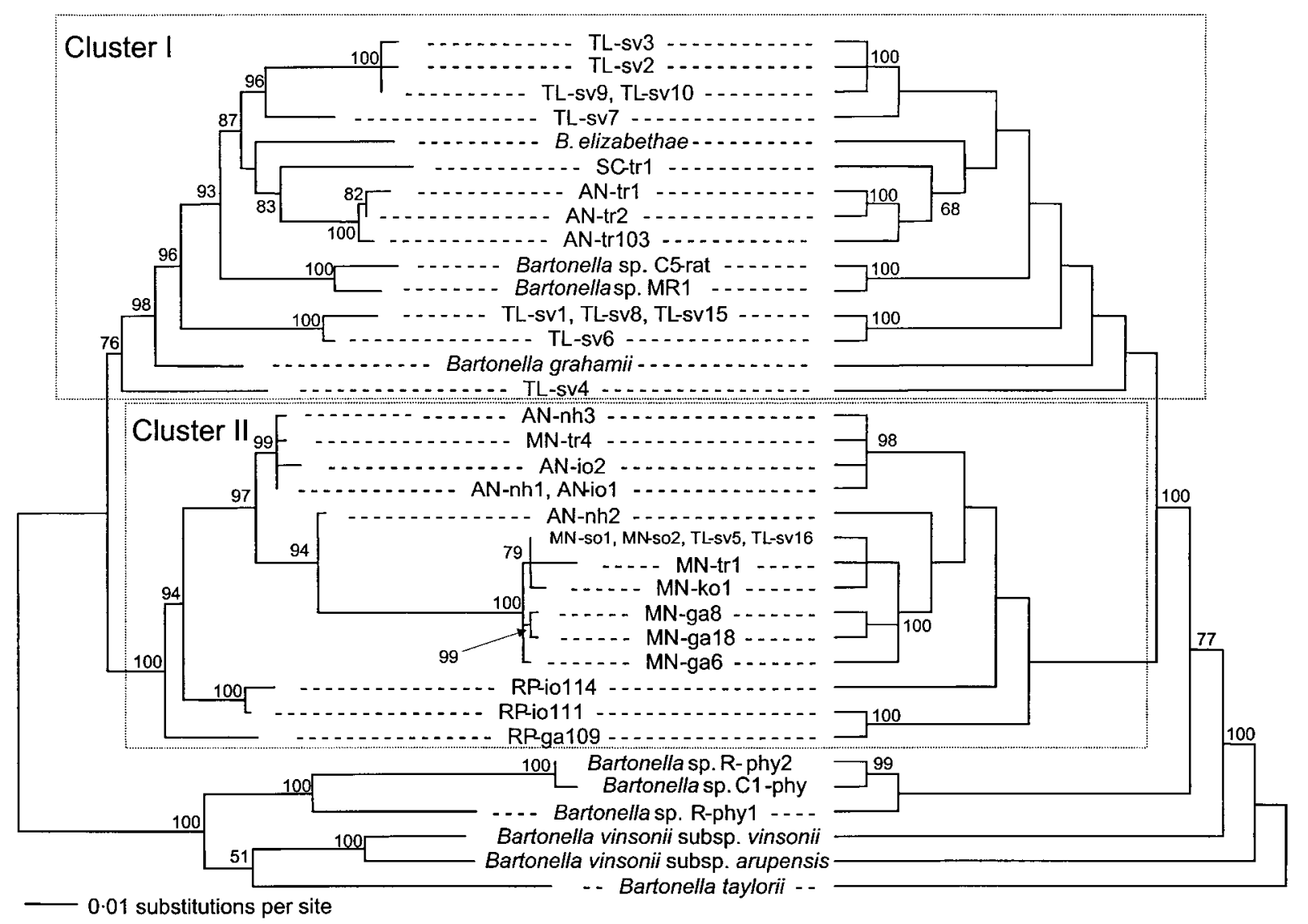

Fig. 2. Phylogenetic trees inferred using a Bayesian (left-hand side) and an MP (right-hand side) approach from alignment of $698 \mathrm{bp} \mathrm{gltA}$ fragments obtained from the SA rodent-associated bartonellae, representatives of closely related Bartonella taxa and other partially characterized Bartonella isolates. Significant bootstrap values are indicated at relevant nodes. Phylogenetic clusters, as defined in the text, are indicated by dashed boxes.

those containing RP-io111, RP-io114 and RP-ga109. Inference of phylogenetic relationships from the $698 \mathrm{bp}$ indicated that the SA rodent bartonellae formed two discrete clusters. Cluster I contained bartonellae infecting A. namaquensis, T. leucogaster and Saccostomus campestris together with bartonellae that are associated with brown rats worldwide (B. elizabethae, C5-rat and MR1) and European woodland rodents (B. grahamii). Cluster II contained exclusively SA rodent-associated bartonellae, including all isolates from $M$. natalensis or R. pumilio (Table 2) together with some of the isolates obtained from A. namaquensis and T. leucogaster (Table 2). Within this cluster, most of the isolates obtained from M. natalensis grouped together (with the exception of MN-tr4). Although the three isolates obtained from $R$. pumilio did not reliably group together, they were all outliers within cluster II, demonstrating clear divergence from the other members of the cluster (Fig. 2).

\section{Distribution of inter-gltA sequence dissimilarity score frequencies}

The 406 pairwise gltA sequence dissimilarity scores determined among cluster I and II members are presented in a matrix (see supplementary table available in IJSEM Online) and ranged from 1 to $63 \mathrm{bp}$. The frequencies of different dissimilarity scores within this range varied and examination of this variation (Fig. 3) suggested that these frequencies fell into a bimodal distribution made up of a smaller mode of frequencies between 1 and $15 \mathrm{bp}$ dissimilarity and a far larger mode of frequencies between approximately 20 and 63 bp dissimilarity.

\section{DISCUSSION}

This is the first published study confirming the presence of bartonellae in rodents endemic to Southern Africa and the first to investigate the diversity of organisms associated with these infections across the entire African continent. The presence and dynamics of Bartonella infections of Psammomys obesus (fat sand rats) in Tunisia have been previously reported (Fichet-Calvet et al., 2000), but the identities of these bartonellae have yet to be published. Although our observations in relation to rodents are new, several studies reporting on Bartonella species currently recognized as human pathogens in Africa have been 


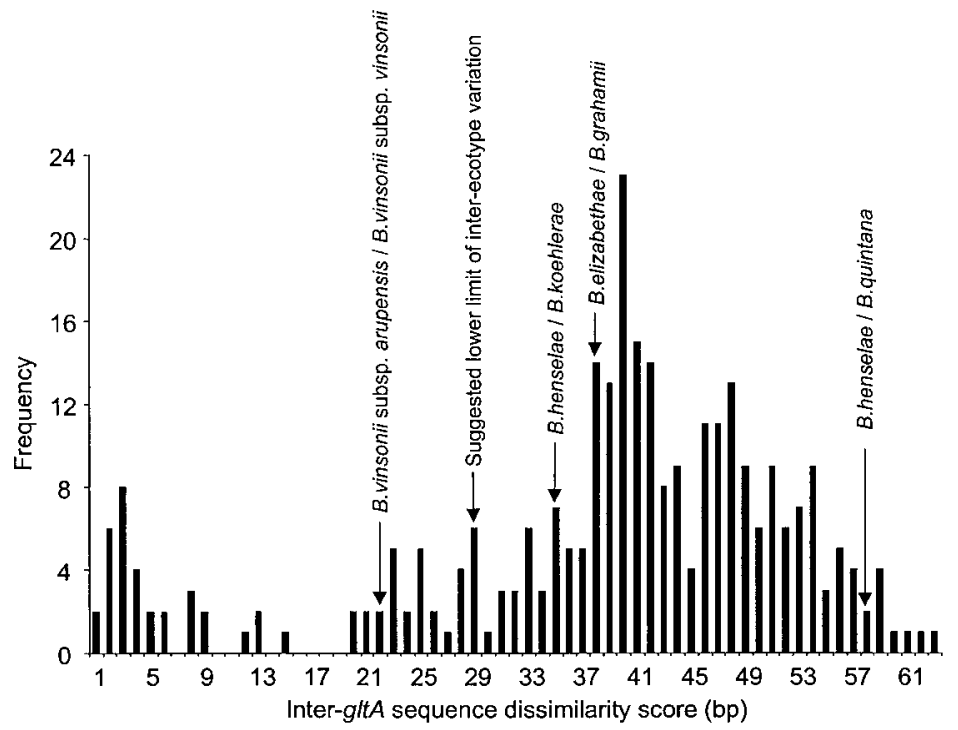

Fig. 3. Bimodal distribution of intersequence dissimilarity score frequencies, as measured by pairwise comparison of $698 \mathrm{bp}$ gltA fragments of the SA rodent-associated bartonellae and other representatives of the genus Bartonella. The previously proposed $96 \%$ threshold value (La Scola et al., 2003) that equates to a dissimilarity score of $28 \mathrm{bp}$ is indicated on the distribution. Superimposed on the distribution are intersequence dissimilarity scores between representatives of several established Bartonella taxa. Their addition is relevant to the discussion and demonstrates the range of scores obtained when taxa are compared.

published. Evidence of $B$. henselae infections in cats and humans has been reported (Childs et al., 1995; Raoult et al., 1996; Kelly et al., 1996, 1998; Pretorius et al., 1999; Frean et al., 2002) and a role for Bartonella quintana as a significant cause of louse-borne disease on the continent has also been demonstrated (Raoult et al., 1998).

The detection of bartonellae in SA rodents is not at all unexpected given that infections have been encountered in all reported surveys of rodent populations elsewhere in both the Old and New Worlds. Communities of rodents and insectivores in Europe (Birtles et al., 1994; Holmberg et al., 2003), Asia (Ying et al., 2002) and North and South America (Kosoy et al., 1997; Birtles et al., 1999) have all been shown to maintain bartonella infections. The overall prevalence of infections observed in SA small mammals (44\%) is similar to that reported elsewhere in the world.

The pattern of clustering of the SA rodent bartonellae is noteworthy. Cluster I includes members of a previously recognized cluster of strains associated with species of Old World rats and mice (Rodentia, Muridae, subfamily Murinae) and Old World-inhabiting species of arvicoline voles (Rodentia, Muridae, subfamily Arvicolinae). Our observations extend the host range associated with this cluster to include the Murinae genus Aethomys, the Gerbillinae genus Tatera and the Cricetomyinae genus Saccostomus (pouched mice). As cluster II appears to represent a novel evolutionary lineage within the genus, no Bartonella isolate of African origin shares any specific evolutionary relationship with Bartonella strains associated with hosts endemic to the New World. This observation further supports the hypothesis that bartonellae associated with hosts native to the Old World are phylogenetically distinct from those associated with host species native to the New World (Ellis et al., 1999).

Examination of sequence similarity data and the inferred phylogenies indicated that some degree of host-specificity among the strains examined exists. Cluster I included three groups of closely related isolates that were obtained from a single host species - two of these groups exclusively contained isolates infecting the only Gerbillinae species studied, T. leucogaster, and one group was associated with A. namaquensis. Cluster II included one group dominated by isolates infecting $A$. namaquensis, one group made up of isolates exclusively associated with $M$. natalensis and a less-well-defined group made up of all three isolates recovered from $R$. pumilio. However, against this background of host preference, there was a clear indication that different host species are susceptible to infection by the same Bartonella species, with, for example, isolates obtained from M. natalensis and T. leucogaster yielding the same gltA sequence and an isolate from M. natalensis lying within the cluster II group dominated by isolates from A. namaquensis. Imperfect host specificity of Bartonella species, particularly those associated with rodents, has been frequently observed (Birtles et al., 1994; Kosoy et al., 1999; Holmberg et al., 2003). From the discussion above, it is also apparent that some host species, for example T. leucogaster, are susceptible to infection by phylogenetically distinct strains of Bartonella; this phenomenon has also been previously observed, with, for example, cats being considered hosts to three Bartonella species (Droz et al., 1999). Intriguingly, no evidence of bartonella parasitism was found in the springhare, Pedetes capensis, despite a reasonable-sized sample (16). Although some other mammalian species have also been reported to be free of bartonellae (Heller et al., 1999), Pedetes capensis is the first rodent species in which no evidence of infection can be found. Springhares are the sole members the family Pedetidae within the order Rodentia and as such are evolutionarily quite distinct from the other rodents sampled in this study.

In the context of the current discussion of the role of sequence similarity data in the definition and delineation 
of bacterial species, the distribution of frequencies of intersequence variation observed among the $29698 \mathrm{bp} \mathrm{gltA}$ fragments obtained from the SA rodent-associated bartonellae and four other Bartonella strains within cluster I and II is noteworthy. Bacteria fall into clusters of closely related organisms based on sequence similarity of shared proteinencoding genes, such that the sequence divergence within a cluster is far less than that between strains of different clusters. Theoretical work has suggested that each of these clusters corresponds to an ecologically distinct population (Palys et al., 1997) and empirical support for this theory has been published (Palys et al., 2000). As the clusters recognized by sequence similarity comparisons have an ecological relevance, it is rational to propose that measurement of this parameter should be a major criterion in demarcating bacterial taxa (Lan \& Reeves, 2001). Our data generally support such an approach, with the frequencies of intergltA sequence variation among our isolates forming a bimodal distribution. The first mode comprised variations between specifically related strains (ecotypes), whereas the second mode comprised variations between strains belonging to ecologically distinct populations of bartonellae. When inter-gltA sequence variations between strains designated as belonging to the polyphasically established Bartonella taxa (both species and subspecies) were superimposed on to this distribution, they were observed to lie exclusively within the second mode (Fig. 3), thereby fulfilling the definition of separate ecotypes. Although this observation suggests concurrence between traditionally defined taxonomic delineations and those revealed by gene sequence similarity assessments, the fact that intersequence variation values between Bartonella subspecies lie within the same continuum as those observed between Bartonella species indicates that subspecies cannot be reliably delineated using a sequence similarity-based approach. As a result of this failure, the ecological relevance of the subspecies demarcation among Bartonella taxa must be called into question.

Of particular interest were the handful of sequence variation values between 9 and $15 \mathrm{bp}$. These values may represent outliers within the broadening genetic spectrum of an ecotype that is generated between periodic selection events (Cohen, 2002), but may also result from recombination of alleles from different ecotypes. Examination of the source of these intermediate sequence variation values indicated that they were derived from comparison of AN-nh2 gltA with the very similar $g l t A$ sequences of AN-nh1 and four other isolates that formed a monophyletic group (see Fig. 2). The distribution of the 12 (synonymous) nucleotide differences within the $698 \mathrm{bp}$ alignment of AN-nh1 and AN-nh2 gltAs indicated that 7/12 lay among the first $106 \mathrm{bp}$ and $4 / 12$ lay among the final $43 \mathrm{bp}$. This distribution was markedly different from that observed in pairwise glt $A$ alignments of members of different ecotypes, in which differences were more evenly dispersed. Furthermore, the $5^{\prime}$ and $3^{\prime}$ extremities of the AN-nh2 gltA sequence were very similar to the gltA sequences of members of the monophyletic cluster containing MN-trl and eight other isolates (Fig. 2). These observations suggested that recombination may have played a role in shaping the identity of AN-nh2 gltA. The role of intraspecies recombination in the generation of genetic diversity among $B$. henselae strains has been demonstrated (Iredell et al., 2003), and an extension of the multilocus sequence typing approach used therein to examine the importance of inter-Bartonella species (or interecotype) recombination events would be valuable.

La Scola et al. (2003) recently proposed cut-off sequence similarity values for Bartonella species definition, suggesting that an isolate could be considered as being a novel species if a $327 \mathrm{bp}$ gltA fragment shared $<96.0 \%$ sequence similarity with those of species with validly published names. This cut-off equates to $28 \mathrm{bp}$ of intersequence variation between the $698 \mathrm{bp}$ gltA fragments we examined, well above the lower limit of interecotype variation observed in Fig. 3, and thus it would appear that its application to species delineation is supported by our data. However, paradoxically, our data also demonstrate the shortcomings of using an approach based solely on sequence similarity assessments for species definition. Comparison of the gltA sequence similarities between TL-sv7 and the three isolates AN-tr1, AN-tr2 and AN-tr103 yielded differences of 29, 26 and 28 bp, respectively. Thus, by applying the criterion described above, TL-sv7 is a separate species from ANtr1 and AN-tr103 but its relationship with AN-tr2 is unresolved. However, phylogenetic analysis of these sequences (Fig. 2) indicated that AN-tr1, AN-tr2 and ANtr103 formed a closely related monophyletic group that was divergent from the branch carrying TL-sv7. Thus, as proposed elsewhere (Lan \& Reeves, 2001), a more sensitive ecologically relevant delineation of Bartonella species could be achieved using a combination of sequence similarity analysis and phylogenetics. However, to reiterate the discussion above, such an approach would benefit greatly from a multilocus approach.

\section{ACKNOWLEDGEMENTS}

We thank J. Watson from the Department of Environmental Affairs and Tourism of the Free State province for his help with capturing and identification of rodent species. Thanks also to Dr A. Radford for useful discussions about the exploitation of sequence similarity values for delineating bacterial ecotypes. This study was partially funded by the University of the Free State, Bloemfontein, South Africa. R. J. B. is supported by a Wellcome Trust Medical Microbiology Fellowship. Part of this work was presented at the American Society for Rickettsiology-Bartonella as an Emerging Pathogen Group, Joint Conference, Big Sky, Montana, 18-22 August 2001 (abstract 131).

\section{REFERENCES}

Birtles, R. J. \& Raoult, D. (1996). Comparison of partial citrate synthase gene ( $g l t A)$ sequences for phylogenetic analysis of Bartonella species. Int J Syst Bacteriol 46, 891-897.

Birtles, R. J., Harrison, T. G. \& Molyneux, D. H. (1994). Grahamella in small woodland mammals in the UK: isolation, prevalence and host specificity. Ann Trop Med Parasitol 88, 317-327. 
Birtles, R. J., Canales, J., Ventosilla, P., Alvarez, E., Guerra, H. Llanos-Cuentas, A., Raoult, D., Doshi, N. \& Harrison, T. G. (1999). Survey of Bartonella species infecting intradomicillary animals in the Huayllacallán valley, Ancash, Peru, a region endemic for human bartonellosis. Am J Trop Med Hyg 60, 799-805.

Bown, K. J., Ellis, B. A., Birtles, R. J., Durden, L. A., Lello, J., Begon, M. \& Bennett, M. (2002). New World origins for haemoparasites infecting United Kingdom grey squirrels (Sciurus carolinensis), as revealed by phylogenetic analysis of bartonella infecting squirrel populations in England and the United States. Epidemiol Infect 129, 647-653.

Breitschwerdt, E. B. \& Kordick, D. L. (2000). Bartonella infection in animals: carriership, reservoir potential, pathogenicity, and zoonotic potential for human infection. Clin Microbiol Rev 13, 428-438.

Childs, J. E., Olson, J. G., Wolf, A., Cohen, N., Fakile, Y., Rooney, J. A., Bacellar, F. \& Regnery, R. L. (1995). Prevalence of antibodies to Rochalimaea species (cat-scratch disease agent) in cats. Vet Rec 136, 519-520.

Cohen, F. M. (2002). What are bacterial species? Annu Rev Microbiol 56, 457-487.

Droz, S., Chi, B., Horn, E., Steigerwalt, A. G., Whitney, A. M. \& Brenner, D. J. (1999). Bartonella koehlerae sp. nov., isolated from cats. J Clin Microbiol 37, 1117-1122.

Ellis, B. A., Regnery, R. L., Beati, L. \& 7 other authors (1999). Rats of the genus Rattus are reservoir hosts for pathogenic Bartonella species: an Old World origin for a New World disease? J Infect Dis 180, 220-224.

Fichet-Calvet, E., Jomaa, I., Ben Ismail, R. \& Ashford, R. W. (2000). Patterns of infection of haemoparasites in the fat sand rat, Psammomys obesus, in Tunisia, and effect on the host. Ann Trop Med Parasitol 94, 55-68.

Frean, J., Arndt, S. \& Spencer, D. (2002). High rate of Bartonella henselae infection in HIV-positive outpatients in Johannesburg, South Africa. Trans R Soc Trop Med Hyg 96, 549-550.

Heller, R., Bermond, D., Delacour, G., Boulouis, H. J., Chomel, B. \& Piemont, Y. (1999). Distribution of Bartonella in European wild mammals. In Abstracts of the 1st International Conference on Bartonella as Emerging Pathogens, Tübingen, Germany, 5-7 March 1999.

Holmberg, M., Mills, J. N., McGill, S., Benjamin, G. \& Ellis, B. A. (2003). Bartonella infection in sylvatic small mammals in central Sweden. Epidemiol Infect 130, 149-157.

Houpikian, P. \& Raoult, D. (2001). Molecular phylogeny of the genus Bartonella: what is the current knowledge? FEMS Microbiol Lett 200, 1-7.

Huelsenbeck, J. P. \& Ronquist, F. (2001). MrBayes: Bayesian inference of phylogenetic trees. Bioinformatics 17, 754-755.

Iredell, J., Blanckenberg, D., Arvand, M., Grauling, S., Feil, E. J. \& Birtles, R. J. (2003). Characterisation of the natural population of Bartonella henselae using multi-locus sequence typing. J Clin Microbiol 41, 5071-5079.

Kelly, P. J., Matthewman, L. A., Hayter, D., Downey, S., Wray, K., Bryson, N. R. \& Raoult, D. (1996). Bartonella (Rochalimaea) henselae in southern Africa - evidence for infections in domestic cats and implications for veterinarians. J S Afr Vet Assoc 67, 182-187.

Kelly, P. J., Rooney, J. J. A., Morsten, J. \& Regnery, R. (1998). Bartonella henselae isolated from cats in Zimbabwe. Lancet 351, 1706.

Kordick, D. L., Swaminathan, B., Greene, C. E. \& 11 other authors (1996). Bartonella vinsonii subsp. berkhoffii subsp. nov., isolated from dogs; Bartonella vinsonii subsp. vinsonii; and emended description of Bartonella vinsonii. Int J Syst Bacteriol 46, 704-709.

Kosoy, M. Y., Regnery, R. L., Tzianabos, T., Marston, E. L., Jones, D. C., Green, D., Maupin, G. O., Olson, J. G. \& Childs, J. E. (1997). Distribution, diversity, and host specificity of Bartonella in rodents from the southeastern United States. Am J Trop Med Hyg 57, 578-588.

Kosoy, M. Y., Regnery, R. L., Kosaya, O. I. \& Childs, J. E. (1999). Experimental infection of cotton rats with three naturally occurring Bartonella species. J Wildl Dis 35, 275-284.

Lan, R. \& Reeves, P. R. (2001). When does a clone deserve a name? A perspective on bacterial species based on population genetics. Trends Microbiol 9, 419-424.

La Scola, B., Zeaiter, Z., Khamis, A. \& Raoult, D. (2003). Gene sequence-based criteria for species definition in bacteriology: the Bartonella paradigm. Trends Microbiol 11, 318-321.

Lawson, P. A. \& Collins, M. D. (1996). Description of Bartonella clarridgeiae sp. nov. isolated from a cat of a patient with Bartonella henselae septicemia. Med Microbiol Lett 5, 64-73.

Palys, T., Nakamura, L. K. \& Cohan, F. M. (1997). Discovery and classification of ecological diversity in the bacterial world: the role of DNA sequence data. Int J Syst Bacteriol 47, 1145-1156.

Palys, T., Berger, E., Mitrica, I., Nakamura, L. K. \& Cohan, F. M. (2000). Protein-coding genes as molecular markers for ecologically distinct populations: the case of two Bacillus species. Int J Syst Evol Microbiol 50, 1021-1028.

Pretorius, A.-M., Kelly, P. J., Birtles, R. J. \& Raoult, D. (1999). Isolation of Bartonella henselae from a serologically negative cat in Bloemfontein, South Africa. J S Afr Vet Ass 70, 154-155.

Raoult, D., Fournier, P. E., Drancourt, M. \& 7 other authors (1996). Diagnosis of 22 new cases of Bartonella endocarditis. Ann Intern Med 125, 646-652.

Raoult, D., Ndihokubwayo, J. B., Tissot-Dupont, H., Roux, V., Faugere, B., Abegbinni, R. \& Birtles, R. J. (1998). Outbreak of epidemic typhus associated with trench fever in Burundi. Lancet 352, 353-358.

Stevenson, H. L., Bai, Y., Kosoy, M. Y., Montenieri, J. A., Lowell, J. L., Chu, M. C. \& Gage, K. L. (2003). Detection of novel Bartonella strains and Yersinia pestis in prairie dogs and their fleas (Siphonaptera: Ceratophyllidae and Pulicidae) using multiplex polymerase chain reaction. J Med Entomol 40, 329-337.

Ying, B., Kosoy, M. Y., Maupin, G. O., Tsuchiya, K. R. \& Gage, K. L. (2002). Genetic and ecologic characteristics of Bartonella communities in rodents in Southern China. Am J Trop Med Hyg 66, 622-627. 